\title{
Airborne Tactical Intent-Based Conflict Resolution Capability
}

\author{
David J. Wing ${ }^{1}$ \\ NASA Langley Research Center, Hampton,VA, 23681 \\ Robert A. Vivona ${ }^{2}$ and David A. Roscoe ${ }^{3}$ \\ Engility Corporation, Billerica, MA, 01821
}

\begin{abstract}
Trajectory-based operations with self-separation involve the aircraft taking the primary role in the management of its own trajectory in the presence of other traffic. In this role, the flight crew assumes the responsibility for ensuring that the aircraft remains separated from all other aircraft by at least a minimum separation standard. These operations are enabled by cooperative airborne surveillance and by airborne automation systems that provide essential monitoring and decision support functions for the flight crew. An airborne automation system developed and used by NASA for research investigations of required functionality is the Autonomous Operations Planner. It supports the flight crew in managing their trajectory when responsible for self-separation by providing monitoring and decision support functions for both strategic and tactical flight modes. The paper focuses on the latter of these modes by describing a capability for tactical intent-based conflict resolution and its role in a comprehensive suite of automation functions supporting trajectory-based operations with self-separation.
\end{abstract}

\section{Nomenclature}

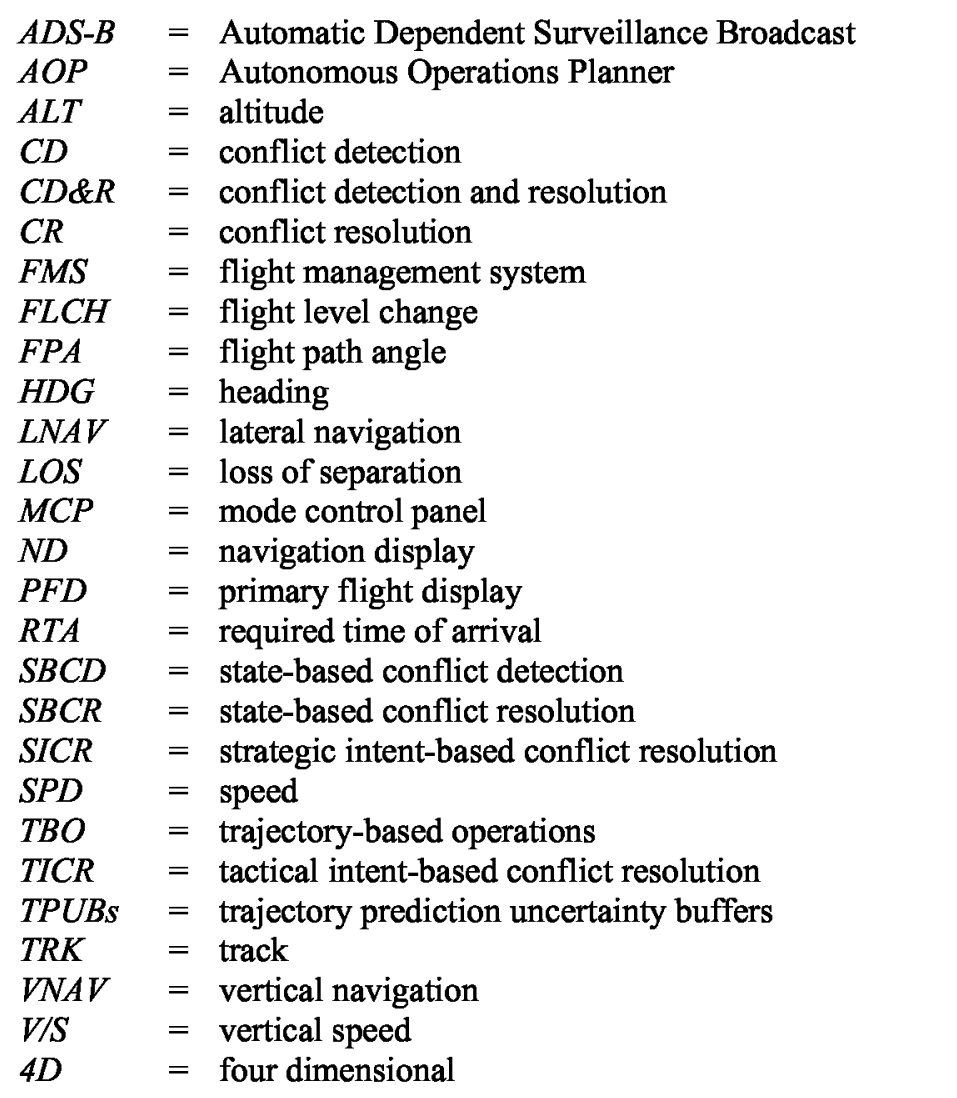

${ }^{1}$ Principal ATM Research Engineer, Crew Systems \& Aviation Operations

${ }^{2}$ Chief Research Engineer, Associate Fellow AIAA

${ }^{3}$ Senior Software Engineer, Member AIAA 


\section{Introduction}

QELF-SEPARATION is an aircraft capability planned for both the US and European concepts of operation for air traffic management. ${ }^{1,2}$ Integrated with trajectory-based operations (TBO), it involves the aircraft taking the primary role in the management of its own trajectory in the presence of other traffic, including assuming the responsibility for ensuring that the aircraft remains separated from all other aircraft by at least a minimum separation standard. The application of TBO with self-separation by multiple aircraft in a shared airspace region results in a distributed control environment in which the responsibility for maintaining separation is distributed among the participating aircraft, rather than regionally centralized in an air traffic controller, as in today's system. Mixed operations are those in which self-separating aircraft share airspace with ground-controlled aircraft.

TBO with self-separation is enabled by cooperative airborne surveillance and by airborne automation systems that provide monitoring and decision support functions for the flight crew. ${ }^{3,4}$ Airborne surveillance technology such as Automatic Dependent Surveillance Broadcast (ADS-B) provides direct electronic broadcast of aircraft state data, enabling aircraft equipped with receivers to know the position and velocity of nearby traffic with high precision and minimal latency. The technology can also provide direct broadcast of limited trajectory data, enabling the receiving aircraft to better predict the future location of the broadcasting aircraft by taking into account planned trajectory change points such as turns and the top-of-descent. This extra information is beneficial in that it allows trajectory management decisions by the flight crew to be more strategic and therefore reduce unnecessary maneuvering. This leads to greater trajectory stability for self-separating aircraft and therefore greater overall system stability and predictability.

An airborne automation system developed and used by NASA for research studies is the Autonomous Operations Planner (AOP). ${ }^{5,6}$ It provides monitoring and decision support functions to the flight crew in managing their trajectory when responsible for self-separation. As a research tool, the AOP provides a high-fidelity design platform to determine what types of decision-support capabilities are necessary to enable TBO with self-separation for a range of complex operational factors. Examples of operational factors that must be accommodated include traffic density that could reach multitudes of today's values, traffic flow structures ranging from well-aligned to randomly oriented trajectories, various amounts of available intent information, traffic conflicts detected early or late, and various uncertainty sources, delays, and failure modes. AOP accommodates these complex operational factors with a functional design intended to emphasize safety.

The development of AOP and its use in research studies have shown that functionality required for TBO with self-separation range from highly strategic to highly tactical. The purpose of the paper is to describe one such function: the tactical intent-based conflict resolution (TICR) function. The description begins in section II by presenting, as context for TICR, an overview of the entire functional structure in AOP that supports TBO with selfseparation. In section III, a detailed discussion follows on the role of tactical maneuvering and TICR. Section IV presents the specific design for TICR. Finally, the flight crew procedures for TICR are discussed in Section V.

\section{AOP Functional Overview}

An understanding of the functional structure of AOP is important to the understanding of TICR's role within it. Three categories of trajectory management functionality are defined, ranging from highly strategic functions to highly tactical functions, each having a specific purpose and role. The categories and associated functions are depicted in Fig. 1 and discussed below.

\section{A. Flexibility Planning}

The most strategic category is flexibility planning. Flexibility planning addresses the exposure of the aircraft to situations that increase the probability of having to change its trajectory or that limit the degrees of freedom of maneuverability should such a change be needed. For example, entering areas of high traffic density would likely increase the probability of encountering a traffic conflict and therefore the likelihood of having to resolve the conflict by making a trajectory change. The assumption is that it is generally preferred by all parties for an aircraft to maintain a stable trajectory without frequent modification. Once the aircraft is immersed in the high traffic density area, conflict probability is now elevated and the aircraft must be prepared to resolve the conflicts, should they arise. However, the high traffic density also reduces the available conflict-free airspace in which to maneuver. 
Reduced conflict-free airspace means the number of viable strategic trajectory changes may be limited. A strategic trajectory change is one that fully reconnects to the destination at the onset of the change and does not include

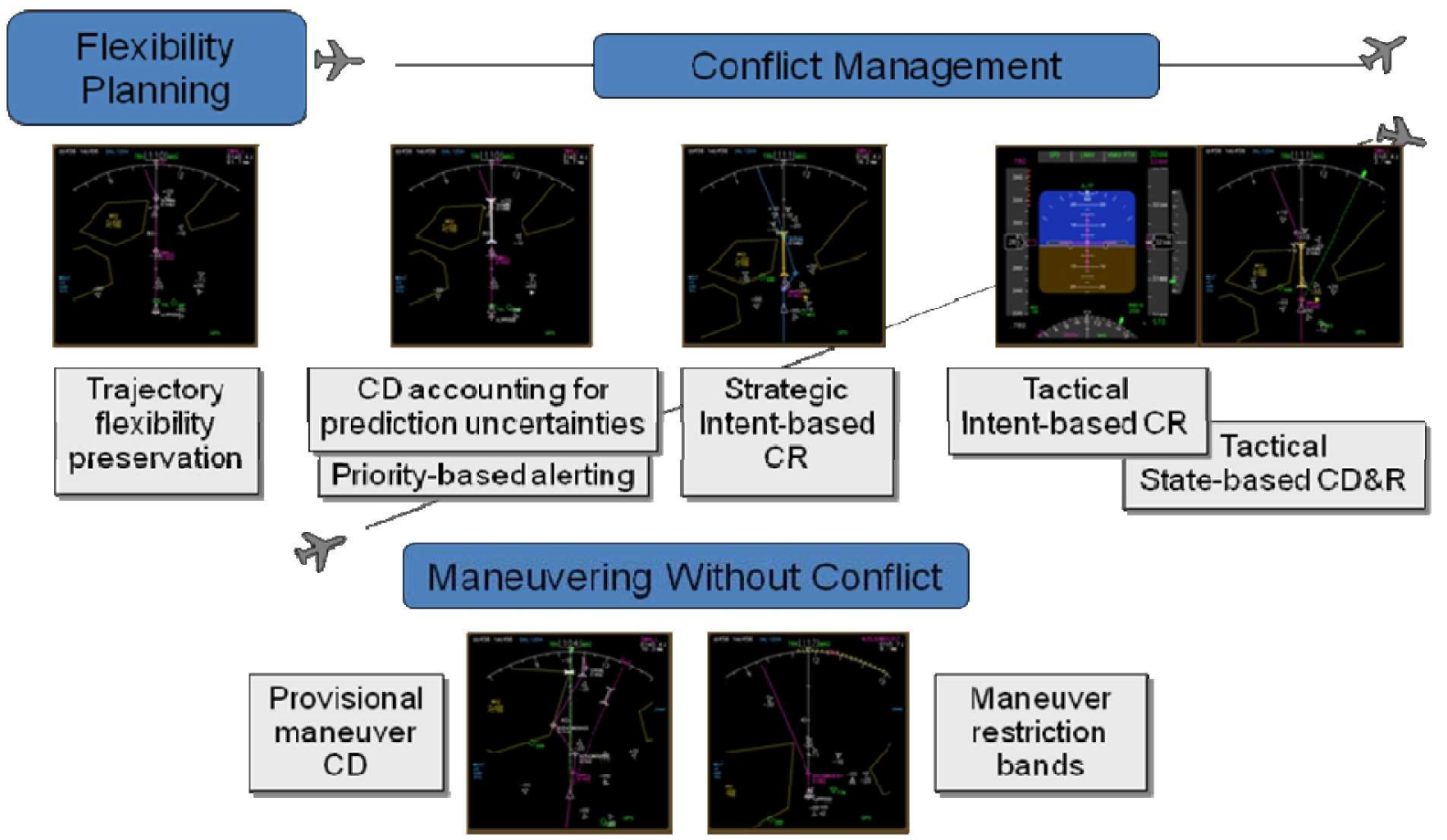

Figure 1. Principal AOP functions for trajectory management and separation provision.

interim, open-ended, tactical maneuvering. The absence of suitable strategic options may require an aircraft to switch to tactical maneuvering, which has less predictability and stability and thus less desirability from a safety viewpoint.

To enable research in minimizing such situations, AOP incorporates the function of trajectory flexibility preservation. ${ }^{7}$ The capability is currently rudimentary, as this cutting-edge research is in an early stage and primarily being conducted outside AOP in a mathematical modeling environment. Flexibility preservation helps mitigate traffic situation complexity by selecting trajectories that can most easily accommodate the possibility of future disturbances such as traffic conflicts and dynamic weather hazards. Its purpose is to reduce the likelihood of conflicts and increase the degrees of freedom available to solve them strategically if they occur. The function computes real-time quantitative metrics for trajectory flexibility: a robustness metric and an adaptability metric. These metrics are based on long-term predictions of the trajectory and its environment (traffic and area hazards). If the predicted flexibility is deemed insufficient, then preemptive trajectory re-planning is initiated to reduce the aircraft's exposure to the complex environment. An expected additional, significant benefit is that overall traffic complexity may be mitigated or even preempted if this function is performed for every TBO aircraft. The method is considered applicable to both self-separating and ground controlled aircraft.

\section{B. Conflict Management}

The second functional category, conflict management, contains both strategic and tactical functions, though the strategic aspect generally does not act as far ahead as flexibility planning. A typical, nominal time horizon for strategic conflict management would be 10 to 20 minutes, whereas flexibility planning may focus well beyond 20 minutes. Conflict management addresses the detection and resolution of conflicts with traffic and well-defined area hazards, as well as appropriate flight crew alerting.

\section{Intent-based Conflict Detection and Alerting}

AOP employs an intent-based conflict detection (CD) function to monitor the currently commanded trajectory for conflicts with traffic aircraft and area hazard polygons. ${ }^{8}$ Because the currently commanded trajectory is not restricted only to the flight management system's (FMS) trajectory, but also accounts for any active tactical flight 
modes and settings established by the flight crew on the Mode Control Panel (MCP), the intent-based CD function is applicable to both strategic and tactical operations. Thus, this function is directly relevant to TICR, as will be discussed later. The nominal look-ahead time for AOP's intent-based CD is 10 minutes. This time horizon has been demonstrated in simulation to provide more than adequate time for alerting and strategic conflict resolution (CR) in highly complex and constrained scenarios and thus may be considered conservative for most situations. Because the CD function's performance is significantly dependent on the accuracy of trajectory predictions of the ownship and the traffic aircraft, a novel approach for accounting for trajectory prediction uncertainties has been devised and employed. Trajectory prediction uncertainty buffers (TPUBs) are applied along each dimension (lateral, vertical, and along-path) of each segment of each trajectory prediction. These buffers effectively extend the linear formulation of a trajectory into a volumetric formulation that encapsulates the anticipated uncertainties of prediction in the three dimensions. TPUBS are shaped and sized based on the particular segment type (e.g., level segments, turns, descents) and can be set to expand or contract at a prescribed rate. The conflict detection function thus searches for intersections between the ownship trajectory's buffered volumes and those of the traffic aircraft. ADSB intent from traffic aircraft is used if available, and this is the preferred mode. In the absence of full intent, AOP uses the best traffic information available, either target state or the state vector, to build traffic trajectories.

A priority-based alerting function applies rules to determine the appropriate time and urgency of alerts for the flight crew. ${ }^{9}$ Right-of-way is the method used to "break the tie" between aircraft in conflict so that generally just one of the aircraft ends up adjusting its trajectory to resolve the conflict. The rule set is based on the maritime rules as adopted and adjusted by aviation for visual flight rules. In addition, rules are included to provide overriding priority to ground-controlled aircraft in mixed operations and to give priority to aircraft in strategic flight modes over those in tactical flight modes. The method employed in AOP is to adjust the time at which conflicts are indicated to the flight crew based on their aircraft's priority in the encounter. Thus, the "give-way aircraft" flight crew will receive indication of the conflict a few minutes prior to the other flight crew. Additional elevations in alert level are also time-shifted. Shifting the conflict indication times and levels between conflicting aircraft generally eliminates synchronous maneuvering by two aircraft, thus eliminating the need for direct coordination of strategic trajectory changes. As will be indicated later, TICR is also subject to similar time-shifting.

\section{Intent-based Conflict Resolution}

Strategic intent-based conflict resolution (SICR) is AOP's primary method for resolving conflicts. ${ }^{10}$ It generates CR options for the flight crew in the form of modifications to the FMS active route. The resolution options provide separation from all aircraft and reconnect to the original route while preserving pre-existing trajectory constraints. SICR manipulates a family of standard trajectory patterns (shapes) to find solutions that match the current situation, as verified by intent-based CD to a 20 minute horizon, and it favors solutions that provide an optimal result. SICR will generally compute both a best vertical solution and a best lateral solution. One is displayed by default, but the pilot can inspect both before selecting one for execution. SICR has been extensively tested in simulation scenarios of extreme traffic density with randomly oriented traffic (all self-separating) and restriction to lateral solutions only. ${ }^{11,12}$ In these exceptionally challenging conditions, it has been found to be very effective with conflicts detected five to 10 minutes away. SICR is the preferred method for conflict management because it compresses all maneuvering actions for managing a conflict into a single strategic decision for the flight crew, while also keeping the aircraft on a four-dimensional (4D) trajectory to the benefit of system predictability.

The tactical complement to SICR is the TICR function. Its role, functional design, and use will be described in sections III $-\mathrm{V}$.

\section{State-based Conflict Detection and Resolution}

Because traffic separation is paramount for safety, AOP's intent-based conflict management functions are complemented by an independent and parallel function set: state-based conflict detection (SBCD) and state-based conflict resolution (SBCR). ${ }^{13}$ SBCD uses only state-vector data for detecting conflicts. By not using intent, statebased approaches are immune to intent-based faults such as non-conformance and blunders. However, because intent of both aircraft are ignored, SBCD is prone to false alerts and missed detections at longer time horizons and in the vicinity beyond planned trajectory changes. SBCD provides "point outs" to the flight crew for near-term statebased conflicts. SBCR overrides the intent-based CR functions only for very short-term state-based conflicts, and it provides one-on-one tactical CR maneuvers to maximize the likelihood of maintaining separation from the nearest conflict. By providing only tactical maneuvers, SBCR design is greatly simplified, its update rate can be very fast, 
and its maneuver advisories can be implicitly coordinated with those of the conflicting aircraft who may also be selfseparating. AOP's state-based functionality has been formally analyzed for correct behavior in all geometries. ${ }^{14}$

AOP's focus is traffic separation, not collision avoidance, and it does not include collision avoidance functionality. Collision avoidance systems such as Traffic alert and Collision Avoidance System (TCAS) and future variants should be considered an independent system with an independent purpose. The interface between AOP and TCAS has not yet been designed but must be carefully considered to ensure there are no adverse interactions or conflicting guidance given to the flight crew. In AOP, it will be assumed that a collision avoidance maneuver takes precedence over the requirement of maintaining traffic separation.

\section{Maneuvering Without Conflict}

The third category of functions supporting TBO with self-separation, Maneuvering Without Conflict, is the direct complement to the second category, Conflict Management. It addresses trajectory changes for all other reasons than resolving a conflict. Besides emergencies, any number of nominal reasons may induce a need to change the trajectory in the vertical or lateral dimension. Examples are turbulence at the current altitude, predicted penetration of hazardous weather, diversion to a new destination, a change in the predicted winds aloft, or a reassignment of scheduled arrival time. In situations such as these, the trajectory change must be preceded by verification that the intended change meets the requirements of separation responsibility and nominally does not induce another aircraft to maneuver.

AOP provides several capabilities to support these requirements. ${ }^{9}$ One such capability is called provisional CD and supports both strategic trajectory changes and tactical maneuvers in "what if" scenarios. The pilot pre-selects the preferred maneuver in either the FMS (strategic) or MCP (tactical) but does not execute it. AOP performs intent-based $\mathrm{CD}$ on the proposed change and provides an indication to the pilot if the change would result in a conflict and at what level (e.g., notification, alert). A clear result means the pilot may execute the maneuver without affecting anyone else. To help the pilot quickly narrow down the choice of tactical maneuvers, an AOP function called Maneuver Restriction Bands displays the CD results of tactical sweeps left/right and above/below the current tactical setting. The sweeping capability is similar to that performed by TICR, which will be discussed in detail in section IV. A third function that supports maneuvering without conflict is AOP's capability to generate a conflictfree path stretch to meet a required time of arrival (RTA). For example, if the aircraft is assigned an RTA that is unable to be met by the RTA capability of the FMS along the active route's lateral path, SICR can be instructed to manipulate its normal trajectory patterns (the same used for CR) to produce a new route that meets the RTA without creating conflicts with traffic or area hazards.

These three categories of functionality were presented to provide the context for TICR. The next section will illustrate that TICR is a critical, if not always primary, function supporting TBO with self-separation.

\section{The Role of Tactical Intent-based Conflict Resolution}

In TBO, with or without self-separation, it is preferred that all aircraft continually operate in a flight mode that supports 4D trajectory prediction and control throughout their flight to the destination. Generally speaking, this flight mode would be lateral navigation (LNAV) and vertical navigation (VNAV) modes engaged, with guidance coupled directly to the active route of the FMS. This flight mode is the design condition for SICR, which provides solutions compatible with this flight mode. Thus, SICR is the primary and preferred method for resolving conflicts, because the aircraft continuously remains in the strategic flight mode throughout the resolution maneuver. This section of the paper discusses the motivation for including the function of TICR, which is to fill two identified gaps in utility provided by SICR and the other functions previously discussed.

1. The first of the two gaps is that normal flight operations cannot always be conducted in a strategic flight mode, that is, in FMS-coupled operations. Although it is the nominal and most desirable state for TBO because predictability is maximized, some operational environments are such that FMS-coupled operations are impractical or too workload intensive for the flight crew. A prime example is airspace impacted by dynamic convective weather. In these situations, decision-making can be highly tactical as the flight crew maneuvers around hazards they identify visually and using onboard weather radar. It is impractical and potentially unsafe to be heads-down, modifying the flight plan, in such a flight environment. If the weather situation is dynamic, repeated re-planning would be necessary to accommodate the ever-changing conditions, which only perpetuates the heads-down operation. In 
addition, there is often insufficient knowledge of the true boundaries of the weather hazard, given the inherent limitations of onboard weather radar (range, shadowing, tilt) or Nexrad weather data (resolution, latency, vertical differentiation), in order to plan a reasonably stable trajectory through the impacted airspace. Flight crews frequently adapt to this situation by flying in tactical guidance modes, enabling them to be more nimble in decision-making and to make the required adjustments without going heads-down.

Therefore, to support conditions where tactical flight modes are operationally necessary, the flight crew must be supported by a primary self-separation capability consistent with tactical flight modes. For instance, if the auto-flight system is set for navigation in "track hold" and "altitude hold" modes, then the CR advisories should be provided as a target track, a target altitude, or a target vertical rate, thereby allowing the flight crew to continue using these tactical auto-flight modes. This requirement could be met by using SBCR, which also provides

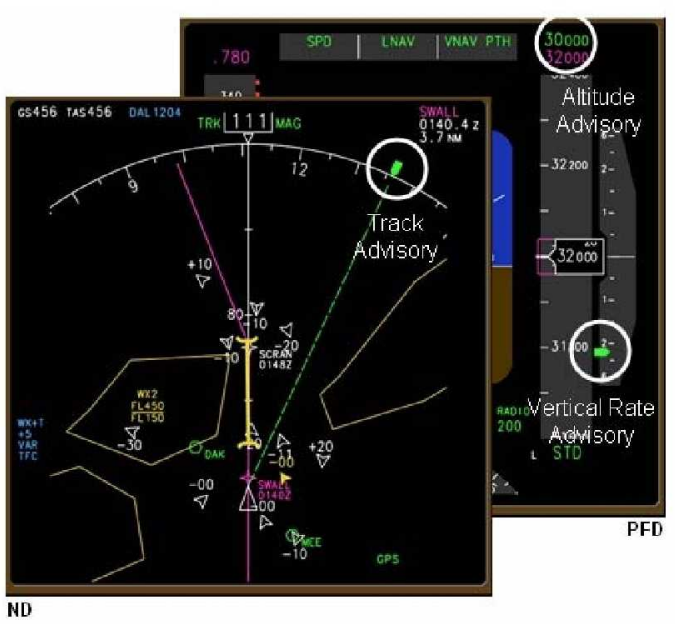

Figure 2. Example of TICR advisories. A track advisory is shown on the Navigation Display (ND), and altitude and vertical rate advisories are shown on the Primary Flight Display (PFD). advisories in tactical form. However, state-based systems have some limiting characteristics, as discussed earlier. They are typically limited to short time horizons, they ignore all intent data, including even limited ownship intent in the form of target states, and they typically account for only one conflict aircraft in conflict resolutions. Thus, the first motivation for developing TICR capability is to support tactical flight modes without giving up some of the advantages of strategic conflict management, such as longer look-ahead horizons, the ability to separate from all nearby traffic, and the use of available intent data from traffic and from the ownship. An example of these advisories produced by TICR is shown in Fig. 2.

2. The second gap that provides motivation for TICR is that a robust safety design should allow for contingencies when the SICR function is unable to produce solutions in a timely manner. Performance testing of the SICR algorithm has shown that such contingencies are very rare but do happen in highly constrained situations and "pop-up" conflicts arising with little time left to solve. ${ }^{11,12}$ When this occurs, an option could be to revert to the SBCR function, although as stated before, much is given up unnecessarily by doing so. Reverting to the TICR function is a better approach. To understand why, it is useful to review the design criteria of the SICR function. SICR is designed to simultaneously achieve multiple objectives in resolving conflicts, shown in Table 1.

Research has shown that AOP's SICR function is very effective at meeting all of these objectives in highly complex scenarios, provided there is sufficient time remaining before the predicted loss of separation (LOS).

Table 1. Functional requirements of the SICR function. Also indicated are the capabilities able to be retained by the TICR and SBCR functions.

\begin{tabular}{|c|l|c|c|c|}
\hline \multicolumn{2}{|l|}{ SICR Functional Requirements } & SICR & TICR & SBCR \\
\hline R1 & $\begin{array}{l}\text { Identify several new trajectories, but at least one, to resolve the } \\
\text { detected conflict }\end{array}$ & $\mathrm{x}$ & $\mathrm{x}$ & $\mathrm{x}$ \\
\hline $\mathrm{R} 2$ & $\begin{array}{l}\text { Allow only trajectories that do not create conflicts with the } \\
\text { remaining traffic }\end{array}$ & $\mathrm{x}$ & $\mathrm{x}$ & - \\
\hline R3 & $\begin{array}{l}\text { Allow only trajectories that remain clear of known airspace } \\
\text { restrictions }\end{array}$ & $\mathrm{x}$ & $\mathrm{x}$ & - \\
\hline R4 & $\begin{array}{l}\text { Allow only trajectories that are within the aircraft performance } \\
\text { envelope }\end{array}$ & $\mathrm{x}$ & $\mathrm{x}$ & - \\
\hline R5 & $\begin{array}{l}\text { Allow only trajectories that retain existing downstream } \\
\text { trajectory constraints, with the exception of an RTA, which can } \\
\text { be relaxed if needed to meet the other requirements }\end{array}$ & $\mathbf{x}$ & - & - \\
\hline R6 & Reconnect to the original route at some downstream point & $\mathbf{x}$ & - & - \\
\hline
\end{tabular}


However, when time to LOS is short, SICR performance may be degraded due to the reduced degrees of freedom and control authority available to meet all of the objectives simultaneously. In this event, the natural mitigation approach to ensuring separation is to begin relaxing the objectives. From Table 1, the SBCR function performs only objective R1, which should be reserved for the last-resort layer of separation management. The TICR function provides an opportunity to retain most of the original objectives. In AOP, the TICR system retains objectives R1 through R4 which involve safety, and it only yields objectives R5 and R6 which do not. In addition, the TICR function in AOP is paired with a subsequent "strategic reconnect" function which, once a stable and conflict-free tactical condition is achieved by TICR, brings in objectives R5 and R6.

Thus, the three functions SICR, TICR, and SBCR create a mutually complementary set of conflict resolution capability. SICR is the workhorse function used in most situations. TICR provides a primary CR capability for tactical flight environments and provides a short-term contingency capability for situations too short-term or constrained for SICR. Finally, SBCR provides an independent and parallel capability focused squarely on resolving the most urgent conflict. The time horizons for integration of these functions, as planned for testing and verification in an upcoming human-in-the-loop simulation, are shown in Table 2. The integration scheme shows that the timing of the conflict indication, associated flight crew action, and CR function available is dependent on whether the aircraft is the give-way or priority aircraft in the encounter, as determined by right-of-way rules. "TICR override" is TICR with a reduced look-ahead horizon matched to the "alert" conflict indication time. The integration scheme for SBCR is under review and is thus not included in the table.

Table 2. Conflict indication, associated flight crew actions, and $C R$ function availability at times associated with give-way and priority aircraft. The right-of-way rule set for a given encounter determines aircraft priority.

\begin{tabular}{|c|c|c|c|c|c|}
\hline \multicolumn{2}{|c|}{ Time to LOS } & \multicolumn{2}{c|}{ Flight Crew } & \multicolumn{2}{c|}{ Resolution Function Available } \\
\hline $\begin{array}{c}\text { Give-way } \\
\text { Aircraft }\end{array}$ & $\begin{array}{c}\text { Priority } \\
\text { Aircraft }\end{array}$ & $\begin{array}{c}\text { Conflict } \\
\text { Indication }\end{array}$ & Action & $\begin{array}{c}\text { Strategic } \\
\text { Flight Mode }\end{array}$ & $\begin{array}{c}\text { Tactical } \\
\text { Flight Mode }\end{array}$ \\
\hline- & 10 minutes & None & None & No CR & No CR \\
\hline 10 minutes & 7 minutes & Notification & Pilot discretion & SICR & TICR \\
\hline 7 minutes & 5 minutes & Alert & Required & SICR & TICR \\
\hline 5 minutes & 4 minutes & Alert & Required & TICR override & TICR override \\
\hline \multicolumn{2}{|c|}{ SBCD: 5 minutes } & Point-out & Pilot discretion & Provisional/FMS & Provisiona1/MCP
\end{tabular}

\section{TICR Functional Design}

This section describes the detailed design of TICR as implemented in AOP. The TICR function produces CR advisories in the form of lateral and vertical target states. A lateral target state is a track (TRK) or heading (HDG), depending on the active lateral flight mode. A vertical target state may be expressed as an altitude (ALT), a vertical speed (V/S), or a flight path angle (FPA). These CR advisories are generated automatically and immediately displayed to the flight crew when available. The advisories are continuously updated using current ownship, traffic, and area hazard information. TICR advisories are provided for all flight modes, including those not supported by SICR.

TICR determines these target-state advisories by sweeping the ownship trajectory through target states on either side of the current state (track, altitude, and vertical rate). Sweeping is performed using the minimum increment selectable on the MCP. Generally, HDG and TRK are selectable in one degree increments, ALT in $100 \mathrm{ft}$ increments, V/S in $100 \mathrm{ft} / \mathrm{min}$ increments, and FPA in 0.1 degree increments. The intent-based CD function is used to identify the first conflict-free condition. The CD function is the same intent-based algorithm used by the SICR function. TICR benefits from high-fidelity ownship trajectory predictions, intent-based traffic trajectory predictions, and uncertainty buffering (TPUBs) for both ownship and traffic. When the aircraft is operated in a tactical flight mode, TICR applies a 12 minute CD time horizon, rather than the 20 minutes SICR uses for resolving conflicts. When TICR is triggered in "override" mode (see Table 2), the CD time horizon is reduced to a time that ensures advisories will clear all conflict indications at the "alert" level.

TICR generally returns the nearest lateral target state to the current track or heading that achieves objectives R1 through R4 in Table 1. In addition, it generally returns the nearest vertical target state to the current altitude, vertical 
speed, or flight path angle that achieves the four objectives. Both the lateral and vertical CR advisories, if available, are displayed to the flight crew. The flight crew may choose which maneuver to fly, because either one will achieve the objectives. If only one maneuver is available, then only one will be returned, and that must be the maneuver flown to ensure achieving the objectives. TICR does not provide a combined advisory that depends on both dimension being executed together.

The process for determining the most appropriate target state advisory is described in the following sub-sections for each active flight mode. In general, the "commanded" value in these descriptions is the guidance value internal to the auto-flight system for a given flight mode. In some cases, the commanded value is established by the flight crew by the settings on the MCP. However, this is not always the case, because the flight crew can enter "preset" values without executing them. AOP maintains an awareness of both the commanded value and the MCP settings.

\section{A. HDG/TRK Hold}

When the lateral flight mode is HDG Hold or TRK Hold, the commanded heading or track value is the current heading or track. The scenario is shown in Fig. 3. TICR is initiated because the commanded trajectory, laterally defined by the commanded HDG/TRK, is in conflict. TICR sweeps in one degree increments starting from the commanded HDG/TRK until a value is found that meets the TICR criteria (Table 1). The total sweep is limited to 60 degrees. If no resolution is found, it sweeps in the opposite direction starting again from the commanded HDG/TRK. The initial direction of sweep (left or right) is in a direction towards the aircraft's destination airport (i.e., the last waypoint in the FMS active route). If the aircraft is currently heading directly towards the destination airport, the sweep direction is arbitrarily chosen to the left. The lateral TICR advisory provided is a target HDG/TRK.

\section{B. HDG/TRK Select}

When the lateral flight mode is HDG/TRK Select, the commanded heading or track value may be different than the current heading or track, and if so, the aircraft is currently turning toward the commanded value. In Fig. 4, the aircraft is commanded to turn left. TICR is initiated because the commanded trajectory, laterally defined by the turn and roll-out on the commanded HDG/TRK, is in conflict. The increment values and limits are the same as HDG/TRK Hold. The order of sweep direction is chosen to bias the result toward the desired (commanded) HDG/TRK. The first sweep is conducted from the commanded HDG/TRK toward the current HDG/TRK (i.e., stopping the turn early). If no resolution is found, the second sweep is conducted from the commanded HDG/TRK to larger turn angles. If no resolution is found, the third sweep is conducted for turns away from the commanded HDG/TRK. The lateral TICR advisory provided is a target HDG/TRK.

\section{LNAV}

When the lateral flight mode is LNAV, TICR, if active, will assume that the flight crew will switch to HDG/TRK Select mode. The behavior will be the same as for HDG/TRK Hold. It ignores the MCP value for HDG/TRK because it may not be properly set.

\section{ALT Hold}

When the vertical flight mode is ALT Hold, the aircraft is level at its current altitude. The scenario is shown in Fig. 5. TICR is initiated because the commanded trajectory, vertically defined by the current altitude, is in conflict. TICR first sweeps altitudes in $100 \mathrm{ft}$ increments in the climb direction, stopping at the service ceiling. If no resolutions are

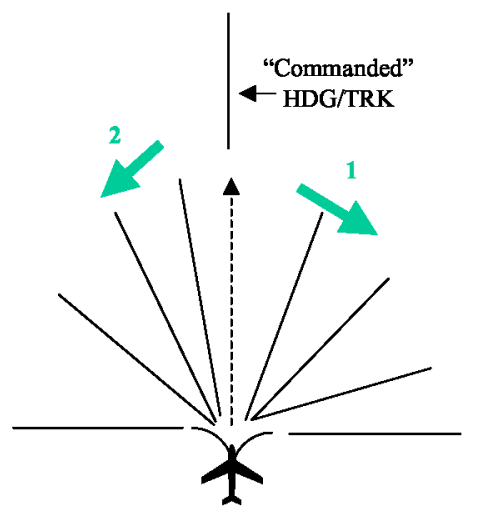

Figure 3. TICR sweep procedure when the active lateral flight mode is HDG/TRK Hold.

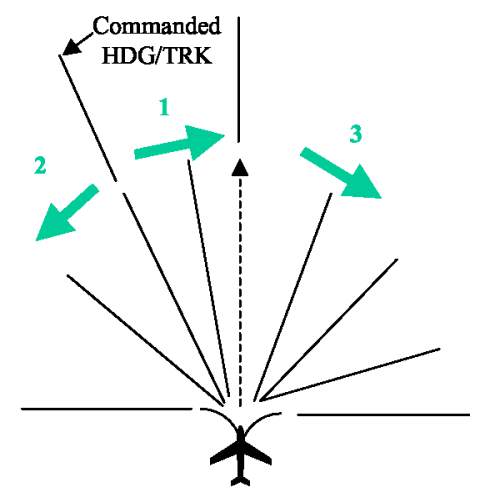

Figure 4. TICR sweep procedure when the active lateral flight mode is HDG/TRK Select.

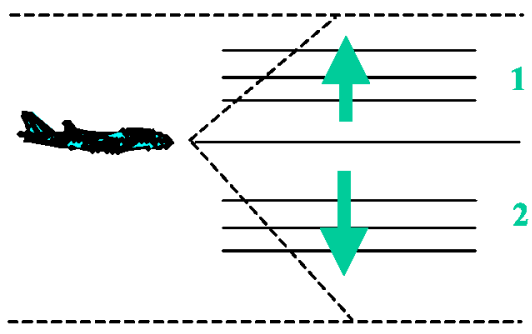

Figure 5. TICR sweep procedure when the active vertical flight mode is ALT Hold. 
found for climbs, TICR sweeps descents, stopping at a defined maximum allowable altitude change. In building the trajectories for each altitude change increment, TICR assumes the flight mode for implementing the change will be a defined procedural climb rate. A near-term future development is to instead assume the Flight Level Change (FLCH) mode is used to change altitudes. The vertical TICR advisory provided is a target altitude with an assumed procedural V/S.

\section{E. V/S or FPA Hold}

V/S and FPA are treated the same way, and so for brevity, only $\mathrm{V} / \mathrm{S}$ is described herein. Four scenarios are possible for V/S Hold.

1. When the V/S Hold value is non-zero and the commanded altitude, defined by the MCP altitude setting, is in the direction of the climb or descent, the aircraft is currently proceeding toward that altitude. These scenarios are shown in Fig. 6. TICR is initiated because the commanded trajectory is in conflict, either along the climb/descent segment or after the level-off. TICR first sweeps increments of $\mathrm{V} / \mathrm{S}$ that accelerate the climb or descent up to a defined maximum allowable vertical rate. If no resolutions are found, then decrements of V/S are swept to a minimum rate of 1000 $\mathrm{ft} / \mathrm{min}$. If no resolutions are found, $\mathrm{V} / \mathrm{S}$ is held constant at the commanded value, and the target altitude is reduced in $100 \mathrm{ft}$ increments to the current altitude (i.e. early level-off). Next, the target altitude is swept beyond the commanded altitude, followed finally by altitudes below the current altitude. The vertical TICR advisory provided is a target $\mathrm{V} / \mathrm{S}$ and a target altitude.

2. When the V/S Hold value is non-zero and the MCP altitude setting is either the current altitude or in the opposite direction of the climb or descent, then the aircraft is climbing or descending and the commanded altitude is non-constraining, i.e., will not terminate the climb or descent. This is not a normal operating condition. The scenario is shown in Fig. 7. TICR is initiated because the commanded trajectory, vertically defined by the commanded V/S, is in conflict. TICR sweeps target altitudes at a constant vertical speed. If the aircraft is in climb, TICR increments the target altitude from the current altitude to the service ceiling, using the commanded V/S. If no resolution is found, TICR decrements the target altitude from the current altitude to a defined maximum descent-change altitude, using a defined standard descent rate. If the aircraft is in descent, TICR decrements the target altitude from the current altitude to the defined maximum descent-change altitude, using the commanded V/S. If no resolution is found, TICR increments the target altitude from the current altitude to the service ceiling, using a defined standard climb rate. The vertical TICR advisory provided is a target $\mathrm{V} / \mathrm{S}$ and a target altitude.

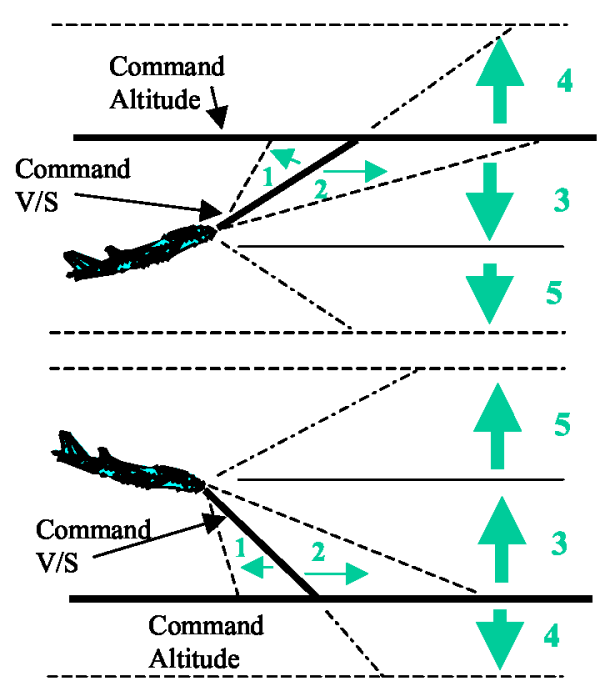

Figure 6. TICR sweep procedure when the active vertical flight mode is V/S (or FPA) Hold and the command altitude is in the direction of vertical flight.

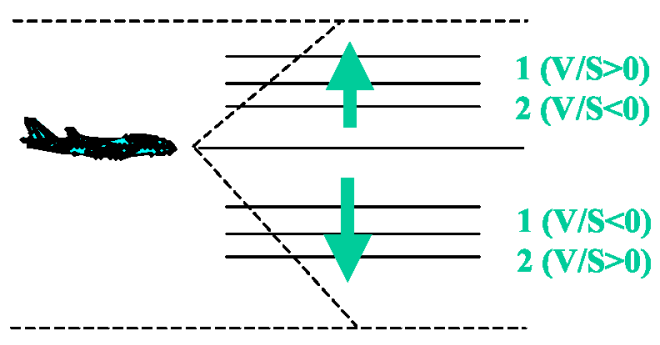

Figure 7. TICR sweep procedure when the active vertical flight mode is V/S (or FPA) Hold and non-zero, and the command altitude is non-constraining.

3. When the V/S Hold value is zero and the MCP altitude setting is not the current altitude, the aircraft is currently flying level, and the flight crew has preset the commanded altitude to a new value. TICR interprets this as a preference for that altitude. The scenario is shown in Fig. 8. TICR is initiated because the commanded trajectory, vertically defined by the commanded V/S (i.e., level at the current altitude), is in conflict. First, TICR attempts to achieve the MCP altitude by sweeping V/S in increments from $1000 \mathrm{ft} / \mathrm{min}$ to a defined maximum climb or descent rate. If no resolution is found, TICR sweeps altitude from the MCP altitude toward the current altitude (i.e. get partway there), using a defined standard climb or descent rate. If no resolution is found, TICR sweeps altitude beyond the MCP altitude. Last, TICR sweeps altitudes in the opposite direction from the MCP altitude, using a defined standard climb or descent rate. The vertical TICR advisory provided is a target V/S and altitude. 


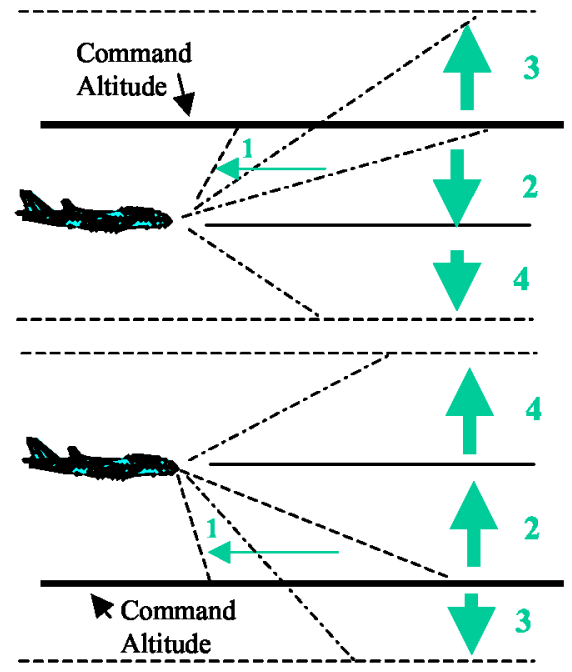

Figure 8. TICR sweep procedure when the active vertical flight mode is V/S (or FPA) Hold $=0$ and the command altitude is not the current altitude.

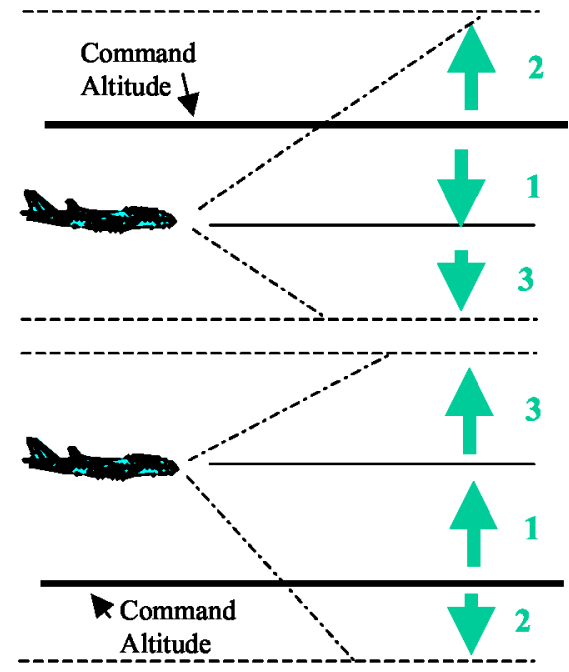

Figure 9. TICR sweep procedure when FLCH is engaged.

4. When the V/S Hold value is zero and MCP altitude is the current altitude, the ALT Hold procedure is used.

\section{F. FLCH}

When the active vertical flight mode is $\mathrm{FLCH}$, the aircraft is currently using FLCH guidance to reach a commanded altitude, defined by the MCP altitude setting. The scenario is shown in Fig. 9. TICR is initiated because the commanded trajectory is in conflict, either along the climb/descent segment or after the level-off. As before, TICR interprets the MCP altitude as the preferred altitude. Using FLCH guidance for the vertical rate, TICR first sweeps altitude from the MCP altitude toward the current altitude. If no resolution is found, TICR sweeps altitude beyond the MCP altitude. Last, TICR sweeps altitudes in the opposite direction from the MCP altitude.

\section{G. VNAV}

When the active vertical flight mode is VNAV (a strategic mode), the tactical resolutions provided by TICR are limited. If the active lateral flight mode is tactical (HDG/TRK Hold or Select), TICR will provide lateral solutions by assuming the current VNAV airspeed (SPD) is maintained if the aircraft is in climb, or by assuming altitude is held constant if the aircraft is in cruise. The approach for VNAV descents is under development. For vertical solutions, TICR sweeps altitude similar to sweep 1 in Fig. 9. Sweeps 2 and 3 are not performed because they are inconsistent with the VNAV path. Currently a defined procedural vertical rate is used, but alternatives such as FLCH and VNAV SPD are being considered as a replacement.

\section{Flight Crew Procedures with TICR}

When TICR advisories are provided, it is either because the aircraft is currently operating in a tactical flight mode or because the "override" condition was triggered. In either case, the flight crew should use tactical settings on the MCP to implement either of the recommended maneuvers, vertical or lateral, depending on the flight crew's assessment of the situation or their preference. Lateral TICR maneuvers are executed by selecting and then executing the target HDG/TRK. Vertical TICR maneuvers are generally executed by first selecting target altitude on the MCP. If no V/S advisory is given, the flight crew should select FLCH to achieve the altitude. If a V/S value is indicated, the flight crew should set the $\mathrm{V} / \mathrm{S}$ to the indicated value to achieve the altitude at the recommended rate.

If both lateral and vertical advisories are provided, either maneuver will independently meet the objectives (see Table 1). Furthermore, the combined maneuver should not be executed, because this maneuver has not been analyzed by TICR. In some situations, only one advisory might be available and should therefore be executed. In the current implementation of TICR, there is no guarantee that at least one advisory is always available. Thus, a 
complete conflict management decision-support system must also include additional functionality focused on resolving the most pressing conflict. In AOP, the state-based functions are intended to meet this requirement.

The flight crew is not obligated to execute either of the TICR advisories. As advisories, they provide information to the flight crew that the indicated maneuvers are conflict-free and may safely be executed. If time and workload permit, the flight crew may alternatively use the "maneuver without conflict" functions to identify other conflict-free and safely-executable maneuvers. This flexibility is both appropriate and necessary because the automation system may not always know the full situation or the intentions of the flight crew. Like all AOP automation functions, TICR is designed to support the human user in a complementary fashion to accomplish the operational objectives of the flight.

\section{Conclusion}

A conflict resolution function suitable for tactical intent-based operations has been developed and is operational in the Autonomous Operations Planner, a NASA research tool for studying the flight deck automation functionality that supports trajectory-based operations with self-separation. The conflict resolution function sweeps through lateral and vertical ranges of operationally viable target states of the ownship aircraft and uses an intent-based conflict detection function to determine the best lateral and vertical solutions that meet four operational objectives. The four objectives are to resolve the conflict, to not create additional conflicts with other traffic, to remain clear of known airspace restrictions, and to remain within the aircraft's performance envelope. The function is intent-based because it uses ownship tactical guidance modes and settings and traffic aircraft broadcast intent, if available, to verify the resolution advisories are free of conflict.

The tactical function supports two primary purposes: to provide primary conflict management support to the flight crew in flight modes other than the fully strategic mode, and to provide a contingency capability in fully strategic modes when the strategic function cannot find a solution or when time is short. The tactical function uses shorter look-ahead horizons and fewer operational objectives than the strategic system in order to increase the likelihood of identifying viable solutions in tactical operations. Thus, the tactical intent-based conflict resolution function is a complementary capability to the primary strategic system supporting trajectory-based operations with self-separation.

\section{References}

${ }^{1}$ Joint Planning and Development Office: Concept of Operations for the Next Generation Air Transportation System, Version 2.0, June 2007. Available for public download from http://www.jpdo.gov/library/NextGen_v2.0.pdf.

${ }^{2}$ Single European Sky ATM Research Consortium: The ATM Target Concept, Milestone Deliverable D3. Document DLM0612-001-02-00. September 2007. Available for public download from

http://www.eurocontrol.int/sesar/gallery/content/public/docs/DLM-0612-001-02-00.pdf.

${ }^{3}$ FAA / Eurocontrol Cooperative R\&D Action Plan 23: The Operational Role of Airborne Surveillance in Separating Aircraft. Deliverable document D3, December 2008. Available for public download from www.icao.int/anb/panels/scrsp/indexp.html.

${ }^{4}$ Wing, D.: A Potentially Useful Role for Airborne Separation in 4D-Trajectory ATM Operations. AIAA-2005-7336.

${ }^{5}$ Ballin, M.; Sharma, V.; Vivona, R.; Johnson, E.; and Ramiscal, E.: A Flight Deck Decision Support Tool for Autonomous Airborne Operations. AIAA-2002-4554.

${ }^{6}$ Karr, D.; Roscoe, D.; and Vivona, R.: An Integrated Flight-Deck Decision-Support Tool in an Autonomous Flight Simulation. AIAA-2004-5261.

${ }^{7}$ Idris, H.; Vivona, R.; Garcia-Chico, J.; and Wing, D.: Trajectory Planning by Preserving Flexibility: Metrics and Analysis. AIAA-2008-7406.

${ }^{8}$ Karr, D. and Vivona, R.: Conflict Detection Using Variable 4D Uncertainty Bounds to Control Missed Alerts. AIAA-20066057.

${ }^{9}$ Krishnamurthy, K.; Wing, D.; and Vivona, R.: Alerting Logic for Multi-Mode CD and Prevention in Autonomous Aircraft Operations. The $23^{\text {rd }}$ Digital Avionics Systems Conference. Volume: 1, pp. 861- 872, 2004.

${ }^{10}$ Vivona, R.; Karr, D.; and Roscoe, D.: Pattern-Based Genetic Algorithm for Airborne Conflict Resolution. AIAA-20066060.

${ }^{11}$ Consiglio, M.; Hoadley, S.; Wing, D.; and Baxley, B.: Safety Performance of Airborne Separation: Preliminary Baseline Testing. AIAA-2007-7739.

${ }^{12}$ Consiglio, M.; Hoadley, S.; Wing, D.; Baxley, B.; and Allen, B.: Impact of Pilot Delay and Non-Responsiveness on the Safety Performance of Airborne Separation. AIAA-2008-8882.

${ }^{13}$ Dowek, G.; Muñoz, C.; and Geser, A.: Tactical Conflict Detection and Resolution in a 3-D Airspace. NASA/CR-2001210853, April 2001. 
${ }^{14}$ Galdino, A.L.; Muñoz, C.; and Ayala, M.: Formal Verification of an Optimal Air Traffic Conflict Resolution and Recovery Algorithm. Proceedings of the $14^{\text {th }}$ Workshop on Logic, Language, Information and Computation, vol. 4576, pp.177-188, July 2007. 